\title{
Design of Camera Application with Physical Sensation Based on Analog on Digital (AoD) Theory
}

\author{
Ziran Fan *, Takayuki Fujimoto *
}

\begin{abstract}
This paper focuses on analog camera, which is making a comeback in recent years after a period of decline since 1990's. After examining the reasons for the popularity of analog cameras, the authors look into the factor of physical feeling users have when operating analog tools, which corresponds with users' physical sensation better than with digital tools. Users get the actual feeling and joy of operating the camera tool through the experience of film-based, analog photographing and physical dial operation, which digital or smartphone cameras cannot easily provide. This paper aims to make the design of smartphone digital content application reflect the physical sensation of using analog tools. We also consider a design method of representing the physical sensation of using analog tools in digital media, taking the example of analog camera. In order to meet users' desire to "get the sensation of using analog tools" and to enable them to have the complete experience of using analog camera on their smartphone, the design method provides functions comparable to those of analog cameras by limiting smartphone functions, and represents the complete film-based photographing experience.
\end{abstract}

Keywords: Analog, Analog Camera, Digital, Interface Design, Application Design.

\section{Introduction}

In general, analog or digital refers to the format of representing information. Analog format represents information in continuous, physical quantities as in analog devices such as clocks and meters. On the other hand, digital format represents information in intermittent numbers [1]. With the advancement of information media such as smartphone, more content design cases are seen in recent years where analog representation of information is used on digital media [2].

This paper focuses on the differences between analog and digital in usage and operating principle of the device, equipment and tools, not from the aspect of representation of information. We will put emphasis on the experience of the users. In this paper, we regard analog as something that has physical existence and works on principles of physics, and digital as something whose electronic mechanism provides data-based content, realizing virtual experience.

* Toyo University, Tokyo, Japan 
Today, digitization has represented analog data on digital media, and enabled users to complete on digital media the tasks that previously were done only with analog tools [3]. Smartphone, in particular, is becoming the most useful tool in everyday life. A generation ago, people used to use analog tools to work and live by. However, now smartphone alone offers everything we need, and it has become modern lifestyle as indicated by the countless number of smartphone applications as well as our own perception. Needless to say, the convenience of smartphone has made our lives easier. However, people would sometimes prefer to use traditional analog tools rather than to make use of digital functionality and convenience even if all analog tools and devices are to be digitized [4]. Paper publications, clocks and records are some of the examples.

We would particularly like to look into a phenomenon that attracted public attention recently. It is the revival of analog camera, or lens with film, which was widely known as disposable camera. It became popular on a global scale in 1980's and 1990's, and then disappeared from the public view with the spread of digital camera. Analog cameras once seemed to have been replaced by digital cameras and smartphone camera applications, but recently they are gaining popularity again especially among young people.

Analog camera and digital camera - or smartphone camera applications - are totally different from each other in terms of functions and usability. The latter is clearly better than the former. Nevertheless, people, especially the young who have not experienced the analog camera fad, love to use the low-functionality, inconvenient analog cameras. This might seem illogical. This paper examines the phenomenon and studies the factor of sensation of using the tool seen in all the analog tools through the example of analog camera. We also consider a digital content design method, which incorporated the sensation of using analog device into digital media.

\section{The Revival Boom of Analog Cameras}

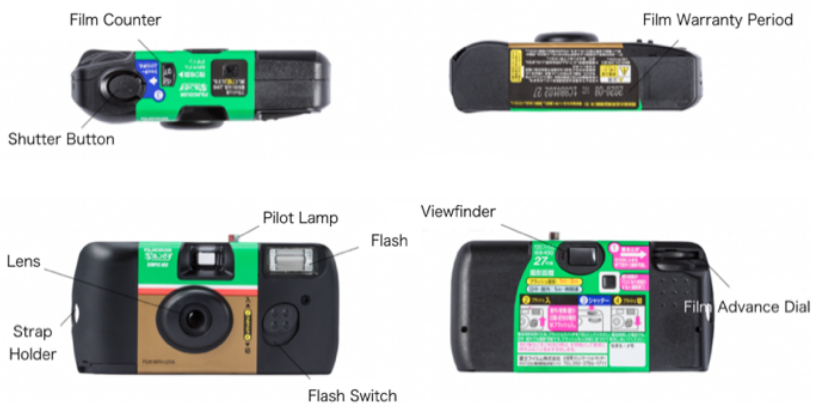

Figure 1: Analog Camera(“写ルンです” from Fujifilm official website)

Analog camera, which is now booming, (Fig 1) is a simple camera with lens attached to the film, just like its official name - "lens with film". Key parts are just film, lens, shutter button and flashlight. There is no need to adjust exposure or focus. Pictures can be taken quite easily by simply pressing the shutter button. Because the product is based on a concept that the lens is attached to the film, users can dispose of it when the film runs out and purchase a new one for an affordable price. For this feature, it has obtained the name of disposable camera and enjoyed much popularity [5]. 
Though disposable cameras were widely used as a daily necessity in 1980's and 1990's, the demand and market size both shrank later on. Recently, many young people take pictures with analog cameras and post them on social media such as Twitter and Instagram, which went viral and created a boom. Many of the users are from the generation who are familiar with smartphones and enjoy using digital media every day.

Some of the users' comments gathered through an internet survey include: "The sense of nostalgia in the photos is great"; "Analog-like photos have a taste like no other"; and "It is actually exciting to be unable to see how the photos would turn out until they have been developed" [6]. In particular, professional photographers stated that it was "a camera that embraced all the pleasures of photographing" [6]. This is because the series of actions - from setting the film, turning the dial to pressing the shutter button to take photos - gives users a feeling of physical and intuitive handling of the camera, which only analog tools can provide. In this way, the photographing experience of using a real camera can be produced.

Analog cameras do not just provide people with a fresh experience of photographing, but also with the sensation of taking pictures using conventional cameras. This realistic sensation enables users to experience the joy of photographing which digital cameras and smartphones can hardly realize [7]. The revival boom of analog cameras indicates the demand for analog device that digital media cannot replace. It also proves that people commonly prefer to have the sensation of physically handling tools produced by their analog, mechanical structure.

\section{Examination of Precedent Examples}

Today, there are many smartphone camera applications whose designs are modeled after analog cameras. Based on the number of application users / number of reviews on AppStore, this paper examines the examples of higher-ranking, typical applications.
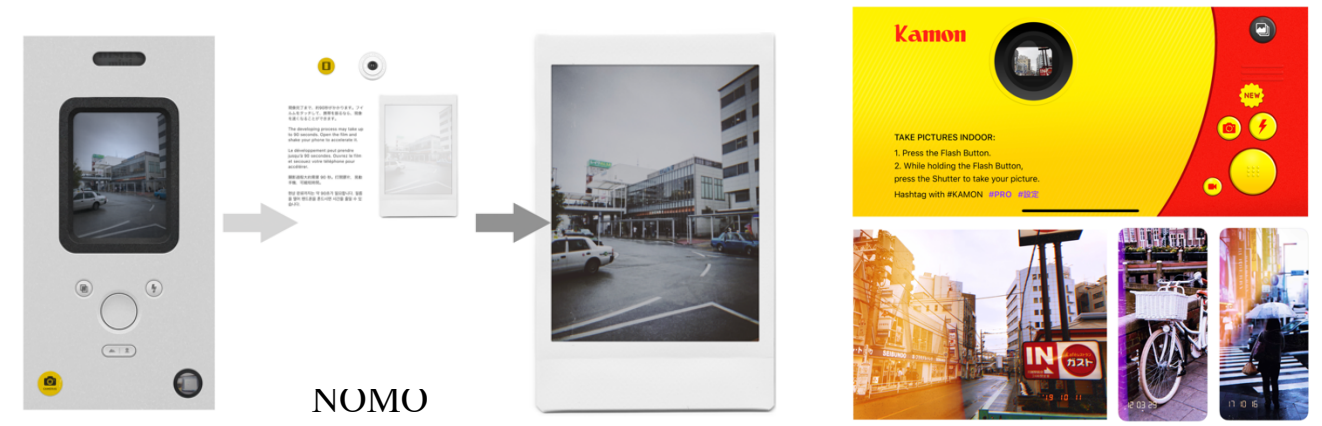

Figure 2: Use of "NOMO" (left) and "Kamon" (right)

"NOMO" features photo developing based on the concept of film. The photos taken with "NOMO" are not immediately displayed and saved as images just like in common smartphone applications, but are gradually displayed, copying the mechanism of photographic film. Moreover, the specifications of analog film and the conventional use of film camera - shaking the developed film - are represented in the application. Users need to wait for 90 seconds to have the pictures developed, but can accelerate this developing process by 
shaking the smartphone.

The action of shaking the smartphone - film - epitomizes the joy of photographing with film camera. Users can experience the analog photographing based on film while taking pictures with smartphone. It also indicated that users prefer the analog concept of shaking the film, which requires time and effort, over the digital method of taking photos in a convenient and efficient way. However, it is also clear that film-based photographing experience is not characterized by the action of shaking the film alone. The analog cameras reviving recently are different from the film cameras shown above in that the photos are not developed in-stantly, but need to be exported from the film and printed on to paper.

Therefore, we examined another precedent. "Kamon" is a camera application with the interface design of analog camera represented on smartphone. The interface design of "Kamon" looks like analog camera, and users can take pictures simply by pressing the shutter button. However, this only reproduces the design of analog camera, and does not deliver the film-based photographing experience or the sensation of using analog camera.

Both examples shown here are modeled after analog cameras, and the analog features of the tool are implemented in the application. However, it is difficult to say that they completely reproduce the experience of using analog camera. It is because the app does not sufficiently provide the sensation of using the "film", which is the core element of analog camera experience. A mere representation of interface design and analog functions does not always deliver the physical sensation of using analog tools.

The sensation of using analog tools provided through interface design and functions as well as the feeling of operating the device with physical reality are indispensable to the "analog-like" experience users want [7][8]. Based on these precedent examples and the concept of reproducing the sensation of physically operating analog tools, we consider in this paper a design method of representing analog camera on smartphone.

\section{Sensation of Using Analog Cameras}

\subsection{Experience of Photographing with Film}

The most significant feature of analog camera is the mechanism of photographing based on its mechanical structure. Users get the realistic sensation of using the camera through the use of "film", a typical analog instrument [6]. Such sensation can be felt through the apparently tiresome and inconvenient tasks such as setting the film before taking photos, or through the constraints of the number of pictures users can take due to the need for one sheet of film per picture.

In other words, the user experience of analog camera largely depends on the sensation of using the film. It gives users a hands-on experience of "using tools" better than digital camera or smartphone camera application, where picture-taking can be done by just pressing the shutter button. The physical sensation of moving objects, rotating the parts or touching them is an ele-ment that is lacking in digital devices like smartphone [9]. Digital cameras may partly retain the traditional operating method of analog cameras compared to smartphones, but they cannot offer the joy of photographing with film as much as analog cameras do.

Film is not just a medium of recording photos, but it functions as an operating device to take pictures. It also affects the quality of photographing experience in addition to enhancing the sensation of using cameras. Those operations and procedures bring reality and enjoyment of 
photographing experience. Especially with analog cameras, users cannot see the photos instantly after taking pictures. Developing photos to paper is the only way to do so. Paperbased photo developing does not only improve the enjoyment of photographing but also raises users' consciousness about the value of each photo.

In this way, film draws a clear distinction between film-based photographing and picture taking with digital camera or smartphone. Film-based photographing also plays an important role in building user experience of camera as an analog tool.

\subsection{Operational Feel of Dial}

You need to operate the film setting dial to take photos by film (Fig 3). The film would be set by turning the dial all the way and users have to turn it every time before photographing.

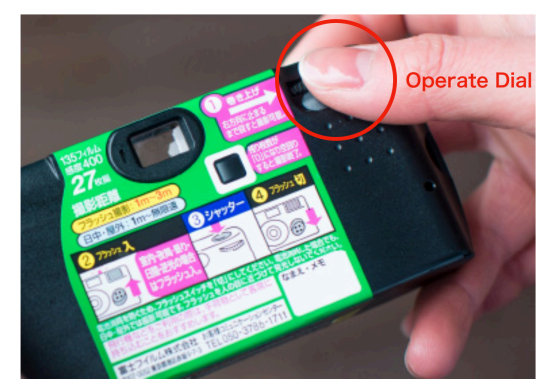

Figure 3: Film setting dial

Regarding current digital cameras and smartphone camera applications, the photos can be automatically taken if users keep pressing the shutter button. On the other hand, as for the analog cameras, setting film by turning the dial with a little time and efforts every time for photographing might be thought as the tiresome task for present generation. However, according to the survey, such tiresome task produces the reality of using a tool of camera and improves the experience of photographing when people take photos.

It is not just because the photographing method of turning the dial, which is different from the usual shooting action of pressing the button, provides users freshness. The physical and tactile sensation of operating the dial, a tangible part of camera, relates to the experience of the analog photographing. In other words, the process of taking photos by turning the dial and setting the film condenses the experience of handling the camera.

The physical and tactile sensation of turning the dial is more suitable for the human somatic sensation than digitized operations. This kind of phenomena have been also demonstrated by many various instances. For example, people prefer reading paper publications because of the sensation of turning pages with the physical touch of paper, rather than turning "pages" by sliding digitized book content on the device screen such as electronic books and smartphone book applications [10]. Moreover, people perceive the feeling of value and attachment better about music with CDs or vinyl records than downloaded data.

The operational sensation produced by the analog-like action of turning the dial also enhances the work efficiency of photographing. Blank time occurs in photographing every time users turn the dial in the intervals of shooting takes. As the scientific demonstration has shown taking a rest between the works leads to the improvement of the work efficiency, setting 
interval in the continuous works raise the efficiency of the works. As on the same context, the operation of turning the dial to set film means the temporary rest of photographing that is practically the "reset" which allows users to get an overview of the photo shooting calmly.

This research aims to reproduce the film system and the sensation of turning the dial, which are the intrinsic components of the analog cameras to construct the unique experience and usability of analog photographing. The purpose is to design the camera application incorporating the analog physical sensation into digital content and to realize a new user experience in the use of digital content.

\section{Design Method}

\subsection{Analog on Digital (AoD) Design Theory}

The design method proposed in this paper is based on the Analog on Digital (AoD) design theory. As T. Fujimoto discusses (2018), AoD theory refers to the idea that incorporates the analog features and factors into content on digital devices and designs/utilizes the digital content on the analog approaches [11]. This idea is also in a common conception with information design theory [12][13].

AoD theory is opposite to the idea of IoT (Internet of Things), which is developing recently. The former emphasizes the user's realistic experience of using tools itself, and the latter values the practicability of technologies and the convenience of the users in case of that they actually utilize those technologies. The goal of IoT is the information network connecting everything to the Internet, in the other words, it aims the complete digitization of the lifestyles in societies [14].

The idea of AoD is not a very new concept. There are many instances representing that idea in our daily lives. Especially the consideration of "human sensation of using tools" emphasized in AoD is valued in the field of entertainment such as the game industry. For example, Nintendo, a famous game company, is positively incorporating the "physical sensation of analog" into the development of game content and hardware.

The novelty of AoD lies in the establishment of the systemized idea organizing the phenomena thought as ordinary before, rather than in the proposal of the idea itself. Among of the numerous design methods, the significance of AoD theory is the attempt to implement the natural-like experience, which is more suitable for human sensation, in the case of people's using digital content by overlooking the content design from the users' point of views and also reproducing the sensory reality of using the very tools [11][15]. In this respect, AoD theory might be thought as the restoration of the old technology, however, the focus of AoD is not on the technology itself but the end-users, who ultimately use technologies [16][17].

AoD theory is also different from the ideas of "usability" or "user-friendly", which are frequently advocated in interface design in recent, and the importance of AoD theory is placed on the "experience of handling tools that people want to use" instead of just providing the "easeof-use" experience of tools [7][11]. As mentioned above, "something easy to use" does not always fit with "something that users want to use."

Based on the design theory of AoD, this paper proposes the method to realize the physical sensation that analog tools have on digital media by presenting the instance of analog camera and demonstrates the practicability of AoD theory to consider its possibility. 


\subsection{Design of Application}

The application proposed in this paper targets iPhones provided by Apple Inc.. The outline of application is shown in fig 4 . The design principle attempts to completely reproduce the experience of operating analog cameras, and it is performed on smartphones not only by the interface design but also by the film-based photographing experience and the physical sensation of camera that depends on the dial. Especially, to provide the enjoyment of operating the very analog cameras, the application ventures to lower the functions of smartphone.

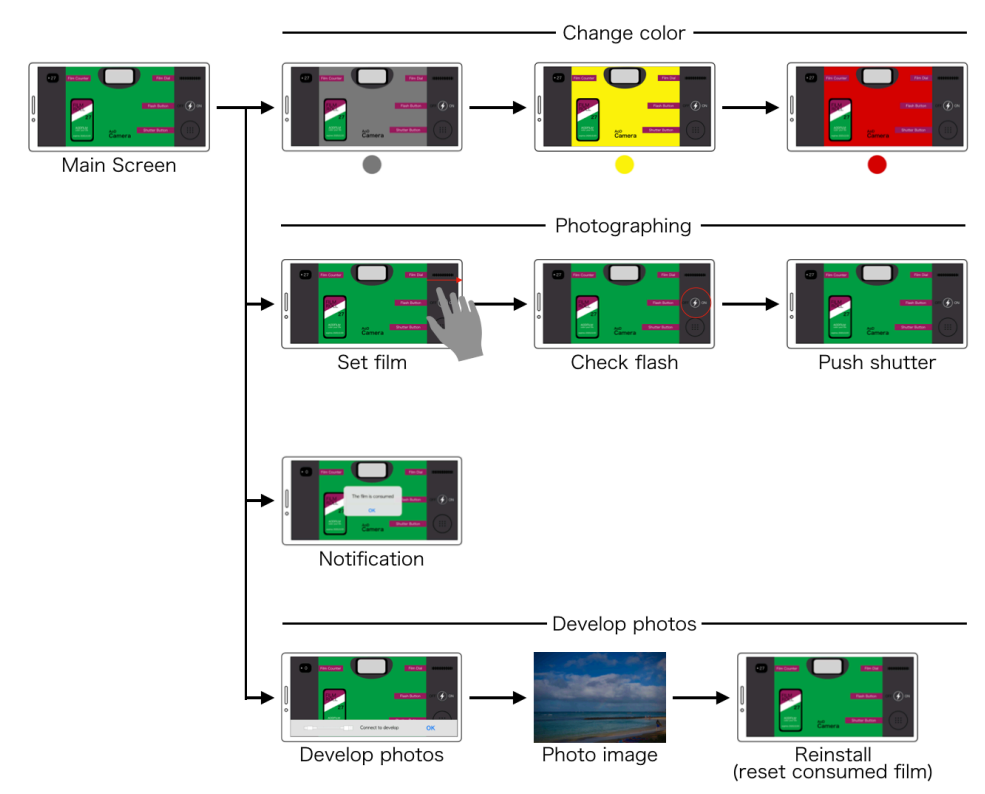

Figure 4: Transition diagram of application

In short, the proposed application turns a smartphone itself to an analog camera, instead of being a digital camera application that has some analog-like effects. Today, people use smartphones as the versatile tools in the daily lives, and this paper aims to add a new usage of analog camera to the diverse uses. Without carry analog cameras, users can enjoy the photographing experience of analog camera with their smartphones, which they always have.

The biggest difference between this application and the precedent examples is the concept design for a camera application like analog camera or the complete reproduction of the analog camera itself. This paper does not deny the necessities of digital camera applications and the ultimate purpose is to represent the physical sensation of operating analog tools on digital way. The most efficient way to realize that is the complete reproduction of the original analog tool.

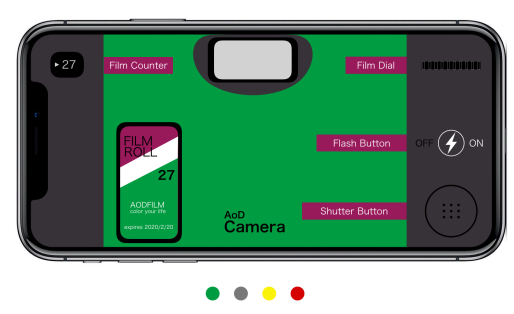

Figure 5: Interface of application 
The interface of application (fig 5) is modeled on the conventional analog cameras. The system provides users with the different color variations for their needs. Regarding the interface design, to accurately reproduce the system of the analog camera, the parts of film, film advance dial, film counter, view finder, flash button and shutter button are designed according to the real analog camera. The usage of application is also resembled with the original analog camera that provides the photographing experience: just pressing the shutter button to take photos. There are no functions provided by general camera applications such as picture effects, automatic continuous shooting and retouching.

The film function is important in the design of the application. The previous camera applications shown in precedent examples are not perfect in respect of implementation of the film function. In many cases, they represent film just as the interface design, which does not practically function, and users cannot obtain the photographing experience by operating the film. This system designs the complete film-based photographing method, which provides a series of the analog experiences from setting film, consuming film sheets to turning the dial on smartphones.

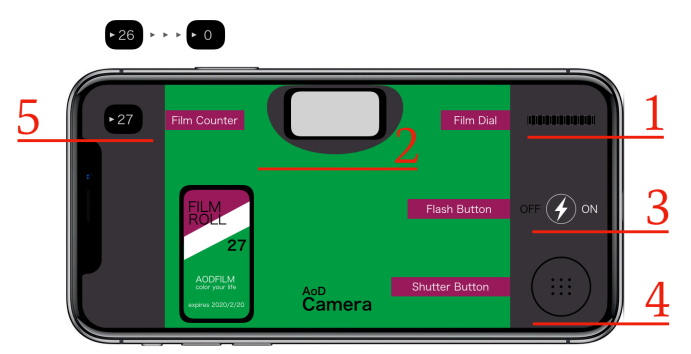

Figure 6: Operation procedure

Fig 6 shows the operation procedure of the application in numerical order. Number 1 indicates the film advance dial and you need to turn the dial to set the film every time before photographing. You can check the object through the view finder (number 2) and change the setting of flash (number 3 ) depending on the shooting situation. Then, you are to press the shutter button (number 4) to take photos. One sheet of film will be consumed every time you take a photo and the number of the film counter (number 5) will decrease.

The function of the film advance dial plays an important role for constructing the whole operational experience of the application. The physical operation of turning the dial does not only provide users with the analog sensory sensation but also evokes the enjoyment of analog photographing. Because the action of turning dial is related to some users' previous experiences of photographing, and also the action itself produces the reality of operating tools.
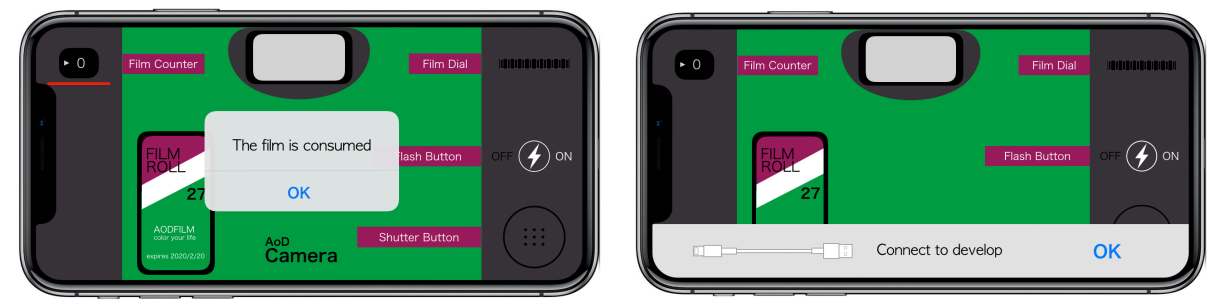

Figure 7: Functions based on the characteristic of the film 
Today, people can take photos freely without concerning about the memory storage on smartphones. Because all the photos are saved in the format of data on smartphones and users can remove photos that they do not like at any time, the users' consciousness about each photo is poorer regarding photographing. In short, since the users can easily take photos without any restriction of photographing such as the film system of analog camera, the sense of value for one photo itself would be degraded.

Regarding the application, the sheets of film are set as 27 according to the standard. The notification would be displayed (fig 7) when all the sheets of film are consumed. Then you cannot continue to take photos with the application according to the analog camera system. As well as the real analog camera, the application has the function that users can restart photographing only by reinstalling the application (= purchasing a new camera/film again).

Moreover, as the design based on the film characteristic, users cannot see the photos taken with the application unless they are developed in paper. When you start the application with the connection of Bluetooth or WiFi to the printer, the option of "develop the pictures" will be available (fig 7). The data of photos will be removed from smartphone once they are developed so that users can see/keep photos only as paper. It is the attempt to provide the consistent analog experience from photographing to seeing (developing) photos by the design of the application.
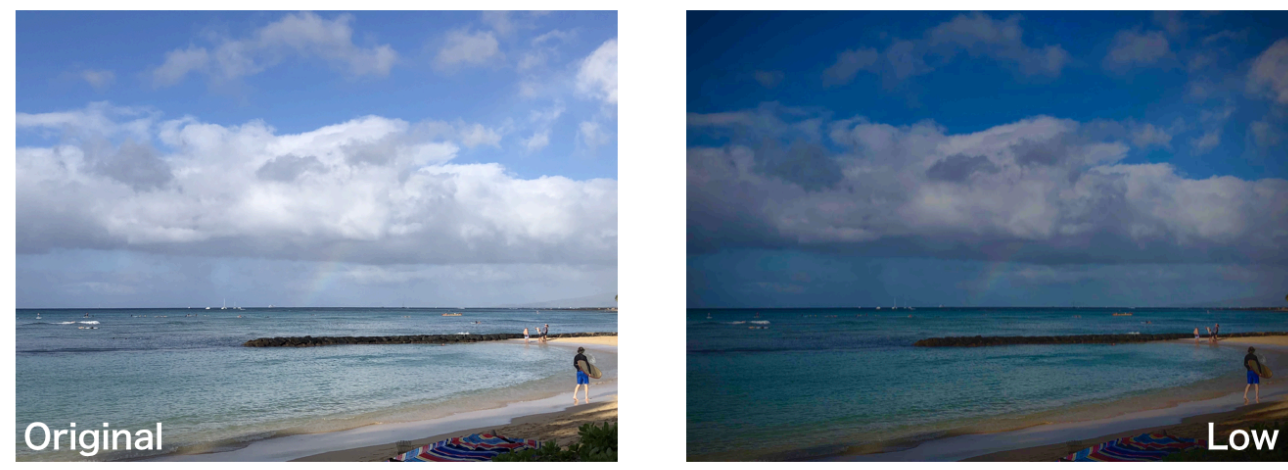

Figure 8: Image of photograph

The designed application does not provide the function to process the pictures for analog look like analog film camera applications mentioned as precedent examples. It provides the pictures that has the image quality as same as ones taken by the analog cameras (fig 8). By intentionally lowering the quality of camera functions of smartphones, i.e. restricting smartphones' performance to be same as analog cameras', the application realizes the same quality of photographing experiences with the real photographing experiences They are different from just the retro experiences. The design of faithful reproduction based on the analog contrivance mechanism aims to improve the photographing experiences and meet users' needs for the use of analog-style objects. Specifically, it incorporates the analog and physical operational feel into digital media, with which the users' sense of value for content tend to fade due to its high convenience.

\subsection{Evaluation}

The proposed application indicated a new content design principle that realizes the experience of operating analog tools on digital media. The attempt was to consider the operational reality of 
analog camera from the aspect of film contrivance and reproduced the film-based photographing experience and the physical sensation of handling analog camera on smartphones. It does not only provide the complete experience of photographing of analogstyle cameras on smartphones, but realized a new user experience by incorporating the analog-style design elements on the smartphones as media.

Digital media is rapidly advancing in regards of functionality and convenience. The application proposed in this paper focuses on users' needs, which are located besides the convenience and functionality. It especially specializes in the general phenomenon that humans prefer the physical sensation of analog. To completely reproduce the analog camera experience on smartphones, convenience and functionality of smartphones are designed to be restricted.

Another attempt of the application was to demonstrate that the user experience for content would be improved by the analog-style operations, which are thought as inconvenient and tiresome. Specifically, the application design incorporated the analog-style physical operation of turning the dial and setting the film to take photos and the system demonstrated the relation in which the analog-style physical sensation leads to the improvement of digital photographing experience.In this way, the proposed application design based on AoD theory indicated the importance of the analog-like elements such as sensory reality on the content design. The idea can be applied to the utilization of every kind of tool, contrivance or device, in addition to the application to the use of cameras.

\section{Conclusions}

Regarding present generations, the people who are required to cope with the developing technologies is users rather than specialists. The advent of new technologies changes the lifestyles of people and replace the various "common sense." The development of the next generation technologies being the base of daily lives like IoT, AI and VR are rising one after another and it requires users to digest and adapt to the generated changes.

The application proposed in this paper adopted uses' needs and reflected them for the application design. Those needs are not referred to how to utilize the latest and outstanding functions, but referred to how to experience the physical sensation that conventional analog tools and contrivances have. The instance of analog cameras is a sample demonstrating such physical sensation of analog and this paper examined the general phenomenon seen in this digital generation through the instance and clarified the ordinary demand of humans to the analog-like experiences. The analog-like user experience is produced by the physical sensation condensed in the buttons, dials and the function principle based on the physical presence and the mechanical structure of objects. Especially in the case of analog cameras, the analog-like user experience relies on the photographing method based on the film.

The proposed application reflects the feature of analog sensation and reproduced it on the digital media of smartphones. The design method to represent the analog elements on digital based on AoD theory is not only the concept to design applications, besides, the idea can be applied to all the digital content packages such as smartphone applications. For example, movie sound effects are made by sounds that do not exist in real lives by applying digital technologies so that audiences could perceive the reality when they are watching movies. 
This paper emphasized the importance of physical sensation of analog on content design again and indicated the new developmental possibility for the conventional computing method. We consider verifying the effect of the proposed method from different aspects as the future work. Especially the effectiveness of the proposed idea is the prior subject and we will adopt the data science methods as the examination measure.

The application of AoD theory of designing analog elements on digital is diverged as mentioned above. It is also necessary to examine the related examples and to consider the wider application in the future work. The technology of digital media is advancing daily-basis and the cyclic periods of devices are being shortened; 1 year or below 1 year. This paper designed the application as the subject of the complete reproduction of the physical sensation of operating analog cameras, however, what AoD theory really aims is also the fusion of analog elements and digital advantages, not just the analog-like representation. In short, the application targets the new user experience by incorporating the analog elements into digital media that has high convenience and functionality. For that purpose, the consideration of the efficient utilization of digital technologies and the redesign of analog elements based on the consideration are required.

This paper is an outset and one of the series of that integrated design idea. We would like to apply AoD theory to more instances and enhance the scientific value of the idea, and then develop the studies from the different point of views from "physical sensation of analog" or the "sensory reality" proposed in the current stage. Our vision is also to design and provide technologies that users want to use, instead of being driven by the treatment of technologies forced by the tide of the times.

\section{Acknowledgement}

This work was supported by JSPS KAKENHI Grant Number 17K00730.

\section{References}

[1] K. Ohno, "Considering Digitalized Document and Content in View of Their History." IPSJ SIG technical reports (digital document), vol. 6, no. 6, 2009, pp.1-12; http://ipsj.ixsq.nii.ac.jp $>$ action=pages_view_main

[2] Y. Hashimoto, "The Evolution of Image Recording Media: from Analog to Digital," Bulletin of the Faculty of Informatics for Arts, Shobi University. vol. 5, 2004, pp.29-44.

[3] H. Kitano, "A Study on Modern Types of Representation in Advertising", Departmental Bulletin Paper of Hiroshima University of Economics $50^{\text {th }}$ Anniversary, vol. 1, 2017, pp. 691-732.

[4] M. Shimasaki, "Considering Analog and Digital," The Public Information of Engineering, Kyoto University, vol. 47, 2007.

[5] Memo about the usage of disposable cameras; https://papacame.com. 
[6] NAVER, Internet Survey of Disposable Cameras; https://matome.naver.jp/odai/2145627485659737401.

$>$ (बidZ. Fan and T. Fujimoto, "Proposal of a 'Disposable Camera' App Providing Sensory Reality mof Analog Operation on Smartphone," 2nd Int'l Conf. Interaction Design and Digital Creation / Computing (IDDC 19), 2019.

[8] Z. Fan and T. Fujimoto, "Proposal of a Design Method to Apply the Analog Features to Digital Media", 2018 World Congress in Computer Science, Computer Engineering, and Applied Computing (CSCE'18), 2018.

[9] Z. Fan and T. Fujimoto, "Proposal of a Digital Book Application that Offers Analog-like Usability", The 15th IEEE Transdisciplinary-Oriented Workshop for Emerging Researchers (IEEE Towers 18), 2018, pp.26.

[10] Z. Fan and T. Fujimoto, "Optimization of Analog Representation in Digital Media for Book Apps”, Applied Informatics and Media Design, vol. 1, 2019, pp. 13-18.

[11] T. Fujimoto, "Ideology of AoD: Analog on Digital -Operating Digitized Objects and Experiences with Analog-like Approach-," $7^{\text {th }}$ Inte'l Congress on Advanced Applied Informatics (IIAI AAI 18), 2018.

[12] T. Fujimoto, "Understandability Design: what is 'Information Design'?," The journal of Information Science and Technology Association, vol. 65, 2014, pp. 450-456.

[13] T. Fujimoto, "Toward Information Design 3.0: The Information Design for 'Communicate',” Building Maintenance \& Management, vol. 34, 2013, pp. 42-46.

[14] R. Eto, G. Yoshikawa and G. Imamura, "Smell Data Mining on the Cutting-edge IoT Olfactory Sensor", The $31^{\text {st }}$ Annual Conference of the Japanese Society for Artificial Intelligence (JSAI 17), 2017.

[15] Z. Fan and T. Fujimoto, "Proposal of a Scheduling App Utilizing Time-Perception-Reality in Analog Clocks", $7^{\text {th }}$ Int'1 Congress on Advanced Applied Informatics (IIAI AAI 18), 2018.

[16] Z. Fan and T. Fujimoto, "Method to Control Children's Smartphone Use Based on the Motif of Analog Fuel System”, Int'1 Conf. Science \& Technology Research (ICSTR Bangkok), 2018.

[17] Z. Fan and T. Fujimoto, "Building an Analog Application to Limit Excessive Use of Smartphones", Applied Informatics and Media Design, vol. 1, 2019, pp. 63-68. 